\title{
Andrologie in einer dermatologischen Praxis
}

\author{
Andrology in a Dermatological Practice
}

Bibliografie

DOI 10.1055/s-2007-966352

Akt Dermatol 2007; 33 :

280-282 @ Georg Thieme

Verlag KG Stuttgart · New York ISSN 0340-2541

Korrespondenzadresse Prof. Dr. med. Carl Schirren Friedrich-Kirsten-Str. 25 22391 Hamburg
Auch ein niedergelassener Dermatologe kann andrologisch tätig sein, wiewohl das in den letzten Jahren vor allem aus finanziellen Gründen seltener geworden ist, wie Kollegen berichten. Das beruht vor allem darauf, dass normalerweise das Angebot an andrologischen Patienten schon dehalb gering ist, weil in aller Regel auch heute noch die Frau es ist, welche bei Kinderwunsch den Gynäkologen aufsucht und in sehr vielen Fällen eine Spermaanalyse - und darauf beschränkt man sich - in dessen Praxis erfolgt, ohne dass der Mann selbst untersucht wurde. Das ändert sich allerdings dann, wenn seitens des Frauenarztes eine besondere Beziehung zu einem andrologisch tätigen Dermatologen besteht bzw. der Mann zu einem Urologen überwiesen wird. Schließlich wird als Grund für diese Entwicklung angegeben, dass die Kosten für eine zusätzliche MTA zwecks Spermaanalysen zu hoch sind, wenn nur ein relativ kleiner Bedarf für derartige Spezialuntersuchungen besteht. So verständlich diese Entwicklung auch ist, sie ist zu bedauern, wenn man bedenkt, dass die Andrologie aus der Dermatologie kam und dass der Erwerb andrologischer Kenntnisse in der Weiterbildungsordnung gefordert wird.

Um zu zeigen, in welchem Umfang und mit welchen Resultaten ein niedergelassener Dermatologe andrologisch tätig sein kann, wird nachfolgend auf Daten aus den Jahren 1953 bis 1969 zurückgegriffen. Einzelheiten sind in der Dissertation von Katrin Schirren ausgewertet worden, wobei als Besonderheit zu werten ist, dass es anfangs nicht ganz einfach gewesen ist, die handschriftlich von C. G. Schirren senior dokumentierten Daten bei 1631 Patienten zu lesen, da er selbst in manchen Fällen seine eigene Handschrift nicht mehr entziffern konnte. Das mindert allerdings den Wert der Einzeldaten nicht.

\footnotetext{
* Nach einem Vortrag auf dem ersten Carl-Georg-Schirren Symposium am 9.12.2006 in Kiel.
}

Ein Vergleich der Patientenzahlen in den verschiedenen Jahren von 1953 bis 1969 zeigt, dass ein kontinuierlicher Anstieg zu verzeichnen war, der in den Jahren 1967/68 einen Höchststand von 254 bzw. 251 betrug.

Das Lebensalter dieser andrologischen Patienten lag mit 907 Fällen in einem Alter zwischen 35 - 40 Jahren; lediglich zweimal betrug das Alter mehr als 51 Jahre.

Die Dauer des Kinderwunsches ergibt für 1-2 Jahre 39 Fälle. Erweitert man diesen Zeitraum auf 1 - 3 Jahre, dann kommt man auf 44,6\%.

Erkrankungen in der Vorgeschichte ergaben in $7,5 \%$ eine Leistenbruchoperation, in $5,7 \%$ eine Gonorrhö, 0,6\% eine Syphilis und in 4,5\% einen Hodenhochstand, der in $43,5 \%$ in dieser Gruppe doppelseitig war.

Das besondere Interesse des Praxisinhabers bei der Betreuung seiner andrologischen Patienten galt den Rauchgewohnheiten derselben. Es lag daher nahe, dass er hierauf sein besonderes Augenmerk richtete. So waren 752 Patienten Nichtraucher und 820 Patienten Raucher. Im Allgemeinen wurden 1-20 Zigaretten täglich geraucht. Als eine logische Konsequenz der Überlegungen hinsichtlich eines negativen Einflusses durch das Nikotin auf die Spermatogenese wurde allen Rauchern daher geraten, das Rauchen vollständig einzustellen und sich drei Monate später dann zu einer Kontrolle wieder vorzustellen. Wenn man sich in diesem Zusammenhang die z. Zt. stattfindende Diskussion über den Schutz der Nichtraucher vor Augen hält, dann waren die damaligen Empfehlungen des Seniors durchaus gerechtfertigt.

Die andrologischen Diagnosen aus dieser Zeit sind $\odot$ Tab. 1 zu entnehmen.

Sie entsprechen damit den einschlägigen Literaturangaben.

Wenn man das therapeutische Vorgehen des Seniors aufschlüsselt, dann hat er in $53,4 \%$ keine medikamentöse Therapie eingeleitet und in $28 \%$ 


\begin{tabular}{lr} 
Normozoospermie & $50,6 \%$ \\
\hline Oligozoospermie & $33,0 \%$ \\
\hline Asthenozoospermie & $12,0 \%$ \\
\hline Azoospermie & $2,3 \%$ \\
\hline Postpuberale Leydigzellinsuffizienz & $2,1 \%$ \\
\hline
\end{tabular}

\begin{tabular}{|c|c|c|}
\hline 1969 & 257 & Tab. 2 An- \\
\hline 1970 & 234 & zahl der an- \\
\hline 1971 & 278 & drologischen \\
\hline 1972 & 372 & Patienten in \\
\hline 1973 & 418 & den Jahren \\
\hline 1974 & 490 & $1969-1994$ \\
\hline 1975 & 705 & \\
\hline 1976 & 740 & \\
\hline 1977 & 686 & \\
\hline 1978 & 710 & \\
\hline 1979 & 600 & \\
\hline 1980 & 676 & \\
\hline 1981 & 655 & \\
\hline 1982 & 655 & \\
\hline 1983 & 628 & \\
\hline 1984 & 653 & \\
\hline 1985 & 734 & \\
\hline 1986 & 677 & \\
\hline 1987 & 640 & \\
\hline 1988 & 601 & \\
\hline 1989 & 652 & \\
\hline 1990 & 593 & \\
\hline 1991 & 623 & \\
\hline 1992 & 670 & \\
\hline 1993 & 550 & \\
\hline 1994 & 612 & \\
\hline
\end{tabular}

aufgrund der Diagnosestellung eine medikamentöse Behandlung vorgenommen. In 18,6\% waren keine Angaben verwertbar. Der Hinweis, bei Störungen der Spermaqualität einer Oligozoospermie bzw. Asthenozoospermie das Rauchen völlig einzustel- len, um auf diese Weise den schädlichen Einfluss des Nikotins auszuschalten, ist nicht als „therapeutische Maßnahme“ klassifiziert worden.

Der Eintritt einer Schwangerschaft aufgrund der andrologischen Untersuchung gehört zu den Besonderheiten der andrologischen Tätigkeit. Denn im Allgemeinen ist es der Gynäkologe, der davon erfährt, während der Androloge in der Regel leer ausgeht. Es ist daher als etwas Besonderes zu werten, wenn unter den Patienten mit einer Normozoospermie in 92 Fällen eine Mitteilung über den Eintritt einer Gravidität erfolgte. Im Allgemeinen wird ein solcher Prozentsatz (42,6\%) aus den genannten Gründen nicht erreicht.

Als eine wohl einmalige Beobachtung ist es zu bewerten, dass C. G. S. im Alter von 78 Jahren bei einem bisher kinderlos verheirateten Mann ausschließlich Rundkopfspermatozoen im Ejakulat festgestellt hatte. Die übrigen Werte des Spermas waren „normal“. Er hatte einen derartigen Befund bisher nicht gesehen und fragte daher telefonisch bei mir an, um meine Meinung zu hören. Ich kannte das Vorkommen von Rundkopfspermatozoen im Ejakulat, nicht jedoch die Tatsache, dass ausschließlich diese Sonderform vorhanden war. Daher schlug ich vor, mir diesen Mann nach Hamburg zu schicken, damit ich dann mit dem mir befreundeten Anatomen A. F. Holstein eine genaue Analyse vornehmen könnte. Wir fanden in der Hodenbiopsie mittels der Semidünnschnitt-Technik ein Vorhandensein von Spermatogenese, jedoch zeigten die Spermatiden eine Fehlentwicklung des Akrosoms. Folglich erschienen im Sperma nur akrosomlose Spermatozoen. Damit war dieser Mann zeugungsunfähig und keiner Therapie zugänglich.

Warum bringe ich dieses Beispiel, das so selten ist und daher für einen praktizierenden Dermatologen mit andrologischen Interessen bedeutungslos sein dürfte? Ich meine, dass dieses Beispiel gerade deshalb einer Präsentation wert ist, weil es überzeugend darlegt: Auch der in der Praxis stehende Arzt ist in der Lage, Entdeckungen zu machen, die bis dato an keiner Forschungseinrichtung und keiner Universität gemacht wurden und zu völlig neuen Erkenntnissen der Spermatogenese beim Menschen führen. Man muss offenbar nur die Gabe besitzen,




das Wichtige von dem Unwichtigen zu trennen, Besonderheiten eines klinischen Befundes zu erkennen und die Möglichkeit suchen können, sich an der richtigen Stelle Rat zu holen.

Dieser Befund wurde von uns mit der Bezeichnung „Rundkopfspermatozoen“ versehen, ein Begriff, der bisher in der Literatur in dieser Form nicht bekannt war. Südlich des Mains taufte man ihn in „Globozoospermie“ um, nachdem man in der Vergangenheit ebenfalls $100 \%$ Rundkopfspermatozoen gefunden hatte. In der Zwischenzeit sind 35 Jahre vergangen. Die seinerzeit erhobenen Befunde und deren Deutung sind unbestritten. Geändert hat sich allerdings die damals getroffene Feststellung der absoluten Zeugungsunfähigkeit dieses Mannes. Denn durch die Entwicklung der intracytoplasmatischen Spermatozoen-Injektion (ICSI) ist man heute in der Lage, auch ein Rundkopfspermatozoon mittels einer Injektion in die Eizelle zu verbringen, wodurch es dann zur Gravidität und schließlich zur Geburt eines gesunden Kindes kommen kann. Unter sog. „normalen“ Voraussetzungen ist das Akrosom dafür verantwortlich, dass es zur Penetration in die Eizelle durch das Spermatozoon kommt. Fehlt jedoch das Akrosom, so ist dieser Prozess nicht möglich.

Mit dem Ausscheiden des Seniors aus der Praxis trat J. M. Schirren als neuer Partner in diese Praxis ein, was zu einer Aktivierung auch der andrologischen Tätigkeit führte, die auf der vorher geleisteten Arbeit des Seniors aufbauen konnte. Hier wirkte sich sein Bekanntheitsgrad in Kiel und Schleswig-Holstein segensreich aus. Die Zahlenaufstellung ( $\bullet$ Tab.2) aus den Jahren 1969 - 1994 belegt das überzeugend.

Die medikamentöse Therapie muss sich an den Befunden orientieren, die erhoben wurden. Sie sollte darüber hinaus auch die
Gesamtsituation des kinderlosen Paares berücksichtigen. So ist es durchaus sinnvoll, wenn eine Absprache mit dem behandelnden Frauenarzt herbeigeführt wird, wie überhaupt die andrologisch/gynäkologische Zusammenarbeit der Schlüssel zum Erfolg sein dürfte. Dabei ist u.a. Folgendes zu berücksichtigen:

1. Ein Spermiogramm muss nicht „normal“ sein, damit die Frau schwanger wird.

2. Eine negative Auskunft an das Ehepaar bei pathologischen Spermiogrammwerten mit dem Tenor „Warten wir erstmal die Behandlung des Mannes ab, bevor wir etwas unternehmen" macht das Paar unsicher. Sie ist auch deshalb widersinnig, weil in manchen Fällen allein durch den Gang des Mannes zum Andrologen immer wieder die Frau schwanger geworden ist.

3. Jede operative Maßnahme bei der Frau und beim Mann ist davon abhängig, dass bei dem jeweiligen Partner ein Befund vorliegt, der eine Operation rechtfertigt.

Mein Beitrag sollte dazu dienen, einen Einblick in die Möglichkeiten zu vermitteln, welche auch in freier Praxis auf diesem Sektor vorhanden sind. Dafür bedarf es einer gesonderten Ausbildung im Rahmen der Weiterbildung zum Facharzt vor der Niederlassung und des regelmäßigen Besuches von Fortbildungsveranstaltungen nach der Niederlassung. Alles erfordert viel Zeit, verlangt ein hohes Maß an Einsatzbereitschaft und setzt vor allem eine besondere emotionale Bindung an dieses Spezialgebiet voraus. Max Weber hat dafür die Worte gefunden: „Nichts ist für den Menschen als Menschen etwas wert, was er nicht mit Leidenschaft tun kann.“ 